\title{
The relationship between the clinical symptoms and radiography results of patients with isolated blunt elbow trauma
}

\author{
Mohammad Davood Sharifi ${ }^{1}$, Narges Rashed ${ }^{2}$, Hossein Zakeri ${ }^{1}$, Hamideh Feiz Disfani ${ }^{1}$, Roohie Farzaneh ${ }^{1}$, \\ Amir Masoud Hashemian ${ }^{1}$
}

\begin{abstract}
Introduction: Elbow injuries are amongst the most common complaints reported by the adults and children referring to the trauma emergency units and they account for $2 \%$ to $3 \%$ of the referrals to the emergency units. The majority of these patients are referred to radiography for an examination of fracture, whereas about $30 \%$ to $40 \%$ of these individuals are found having no clear need for radiography in clinical examinations. The present study aims at investigating the relationship between the clinical and fractures' examinations scales and their usability for predicting the elbow bone injuries in patients with blunt elbow joint trauma.

Patient and Method: The study sample volume included the entire patients with blunt elbow joint trauma who had referred to Hasheminejad and Imam Reza (Peace be upon him) Hospitals during the time span from 2014 to 2015 and also had no past records of elbow joint fracture, surgery and deformity. After performing a preliminary examination of the patients' elbow joints which was conducted in the form of extension, supination, pronation, topical tenderness evaluations in such regions as ulnar head, radial head and humerus epicondyle as well as investigation for the existence of ecchymosis and hematoma in the elbow joint region, the patients were subjected to standard lateral and anterior-posterior (AP) radiography; the results of the examinations were recorded in a checklist that had been prepared beforehand. Then, the results of the radiographies were studied based on which the proper treatments were figured out. Afterwards, the results of the radiographies were matched with the preliminary examinations and finally they were all exposed to evaluations so as to come up with a conclusion.

Results: The study sample volume included 188 patients. Based on the fracture type, the logistic regression analysis data indicated that there is a significant relationship between radial head fracture with restriction in supination of the forearm, sensitivity to palpation on the radius bone head, tenderness to topical touching of the ulnar head bone and sensitivity to topical touch of epicondyle of the humerus and that the former can be applied as a predictor for all of the latter signs $(P<0.05)$. Also, the analyses were indicative of the existence of a significant relationship between the ulnar bone head fracture with pronation and supination limitations, sensitivity to palpation of the ulnar head bone $(P<0.05)$. In distal humerus fractures, the clinical sings are predictable in the form of extension restrictions and tenderness to palpation on the radius head $(\mathrm{P}<0.05)$. Logistic regression analysis data were also indicative of a significant relationship between the proximal radial fracture only with supination limitations in elbow joint position $(P<0.05)$ and the combined humerus fractures can be predicted by supination limitations and hematoma in elbow joint $(P>0.05)$. Also, the data analyses showed that there is no significant relationship between the proximal ulnar fracture and any of the clinical signs so the clinical symptoms were found incapable of properly predicting the proximal ulnar fractures $(P>0.05)$.

Conclusion: Based on the data analysis, it seems that some of the clinical scales can be used as parts of acceptable instruments for predicting the elbow joint fractures so that the unnecessary diagnostic radiographies could be prevented.
\end{abstract}

Keywords: extension, supination, pronation, ecchymosis, hematoma, blunt elbow trauma

\section{INTRODUCTION}

Elbow joint fractures and dislocations are inter alia the most common injuries that occur in various ages, especially during childhood and adolescence mostly when playing sports or upon falling down from elevated surfaces as well as simply by falling down or during playing games like tripping when skating or in severe impacts that are inevitable in such games as football (1). The abovementioned incidents can cause various injuries: isolated dislocation of the elbow joint, distal humerus fracture, proximal radius and ulna fractures or a combination of the two (2). Besides these, other

\footnotetext{
1 Department of Emergency Medicine, Faculty of Medicine, Mashhad University of Medical Sciences, Mashhad, Iran.

2 Equally First Author, Mashhad University of Medical Sciences, Mashhad, Iran.
}

Correspondence: Amir Masoud Hashemian

Department of Emergency Medicine, Faculty of Medicine, Mashhad University of Medical Sciences, Mashhad, Iran

Received: 5 Feb 2018, Accepted: 12 April 2018

E-mail: HashemianAM@mums.ac.ir

(C) 2018 by the authors; licensee Modestum Ltd., UK. This article is an open access article distributed under the terms and conditions of the Creative Commons Attribution License (http://creativecommons.org/licenses/by/4.0/). 
types of interior and exterior joint injuries have also been defined that can be accompanied by partial or full-scale lesions (3). Elbow joint dislocation can occur as a single injury or in combination with the other fractures or injuries (4). The elbow injuries are unpleasant in that they cause a loss of joint movements that might be subsequently followed by fibrosis and ossification of the capsule or, better said, ossification of the joint (5). The radial head and neck fractures are prevalent in the forearm (6).

The routine method of treating elbow injuries in emergency units is performing radiography in several various states (at least two views) for the diagnosis of fracture or dislocation (7\&8). But, one has to always consider the fact that the radiographic interpretation of acute trauma in children is rendered difficult due to the emergence of ossification centers in various ages like capitulum in 1 to 2 years of age, medial epicondyle in 5 to 6 years of age, trochlea in 9 to 10 years of age and lateral epicondyle in 9 to 12 years of age, (9).

According to the high occurrence rate of such accidents and their high likelihood in certain age and occupation groups, particularly in professional athletes, the radiographers' investigation through impact test, without considering the intensity, the location and the status of the impact, will not only incur the treatment system with huge losses, it might also be followed by the possible damages resulting from exposing the injured to the a single-time or multiple-time radiation (10).

Although there are scattered prior researches performed on joint distension and range of motion reduction, the general summarization of post-traumatic evidences, especially on ecchymosis and hematoma, is yet to be carried out. Various factors can influence the injuries resulting from trauma regarding the way the injury has been imposed, its intensity, its symptoms and the treatment method of interest. Age, trauma intensity, the method the trauma or pressure has been incurred are amongst the most important factors (11).

Although the patient's age, his or her physical status and a brief description of the way the trauma has been incurred by the elbow joint can generally be helpful in perceiving the intensity of the injury, the absence of special academic criteria for taking diagnostic measures on joint injuries made us to perform a study based on post-traumatic joint status so that the extension, supination, pronation, ecchymosis, hematoma and topical tenderness can be compared as signs of the elbow injuries with the routine findings of the emergency units to finally come up with a method for predicting, hence making proper decisions, regarding the injury incurred. Evidently, if the relationship between any of the aforementioned symptoms with the bone injury or dislocation turns up statistically significant, it can be applied for formulating an assumption regarding the likely bone injuries in patients with blunt elbow trauma. The impact prognostication will be investigated of its usefulness and, in the end, a method will be proposed to make use of the scales for taking elbow radiographs.

\section{PATIENTS AND METHODS}

The present study has been conducted based on a census method. The study sample volume included all of the patients who had referred to emergency and accident department of Mashhad's Imam Reza (PBUH) and Hasheminejad Hospitals for blunt elbow trauma during the time span from 2014 to 2015. The study sample volume was found in an age range from 18 to 70 years of age. The data have been collected based on field and laboratory research and observation as well as interview.

After performing detailed preliminary examinations, conducted in the form of extension, supination, pronation, local tenderness evaluations on radial head and ulnar head regions as well as on humerus epicondyle and also the investigations of the existence of ecchymosis and hematoma in elbow joint, the patients were referred for standard lateral and anterior-posterior (AP) elbow joint radiographies based on which appropriate treatments were suggested.

Then the results of the radiographs were compared with the preliminary diagnosis to reach to a final conclusion.

After the required data were collected and inserted into SPSS, version 21, there was made use of diagrams, statistical tables and data point dispersion and concentration indices to describe the data and logistic regression analysis was employed to analyze the information. In the entire calculations, the significance level was set at $P<0.05$.

\section{Inclusion Criteria}

The entire patients with blunt elbow joint trauma in a range from 18 to 70 years of age were allowed to enter the study. 
Table 1: The relationship between the radius head fracture and the findings of clinical examinations in the patients with blunt elbow trauma

\begin{tabular}{clccc}
\hline Row & Clinical examination & OR & Cl (95\%) & P-value \\
\hline 1 & Extension limitation in elbow joint & 0.50 & $0.19-1.27$ & 0.14 \\
\hline 2 & Pronation limitation in elbow joint & 1.57 & $0.40-6.06$ & 0.51 \\
\hline 3 & Supination limitation in elbow joint & 0.13 & $0.03-0.60$ & 0.008 \\
\hline 4 & Tenderness to topical touch on radial head & 0.05 & $0.01-0.18$ & $<0.001$ \\
\hline 5 & Tenderness to topical touch on ulnar head & 4.97 & $1.39-17.67$ & 0.013 \\
\hline 6 & Tenderness to topical touch on epicondyle of humerus & 9.74 & $1.80-52-68$ & 0.008 \\
\hline 7 & Development of hematoma in elbow joint & 0.86 & $0.28-2.66$ & 0.80 \\
\hline
\end{tabular}

\section{Exclusion Criteria}

1) Patients who expressed their dissatisfaction for taking part in the study.

2) Patients with prior histories of elbow fractures.

3) Patients with prior elbow joint deformities.

4) The patients with prior history of elbow joint surgery.

5) The patients with inflammatory and infectious diseases.

6) The patients with a past history of neuromuscular diseases.

\section{Ethical Considerations}

All the patients were firstly debriefed about the study. Then written consent letters were acquired from them followed by the insertion of the patients' information in the previously prepared forms. No additional costs were imposed on the patients and they were assured that their information will remain confidential.

\section{RESULTS}

The study sample volume included 188 patients who had referred to the emergency units of Mashhad's Imam Reza (PBUH) and Hasheminejad Hospitals in 2014 for blunt elbow trauma. Out of the patients referred to the abovementioned hospitals, 126 individuals (67\%) were male and 62 individuals (33\%) were female. This number of the patients were classified to four groups based on their age group: there were 68 patients, in a range from 15 to 30 years of age, in the first group with a frequency percentage of $36.2 \%$; the second group was comprised of 52 patients, ranging in age from 31 to 43 years of age with a $27.7 \%$ frequency distribution; the third group was consisted of 47 patients in an age range of 44 to 56 years of age with a frequency distribution of $25 \%$ and, lastly, there were 21 patients in the fourth group ranging in age from 57 to 70 years of age and an $11 \%$ frequency distribution. The four groups were also classified to the five following categories in terms of the trauma reasons: vehicle passengers, 5 individuals (2.7\%), motorcycle passengers, 45 individuals (23.9\%), passerby and having an accident with vehicle, 31 individuals (16.5\%), direct hit, 29 individuals (15.4\%) and falling down, 78 individuals (41.5\%).

The entire patients were evaluated and examined. The patients were also categorized in terms of the developed symptoms as follows: extension limitation in elbow joint (104 individuals), pronation limitations in elbow joint (89 individuals), supination limitation in elbow joint (57 individuals), tenderness to topical touch on the radial head (101 individuals), tenderness to topical touch on ulnar head (52 individuals), tenderness to topical touch on humerus epicondyle (28 individuals) and hematoma in elbow joint (38 individuals).

The patients were next referred to radiography unit for lateral and anterior-posterior (AP) elbow joint standard radiographs. The injuries, as evidenced in the radiography images, were divided into radius head fracture, 41 individuals, ulna head fracture, 21 individuals, distal humerus fracture, 14 individuals, proximal radius fracture, 16 individuals, proximal ulna fracture, 7 individuals and combined radioulnar fracture of the humerus, 11 individuals.

There was made use of logistic regression tests in a 0.05 significance level to investigate the relationship between the seven bimodal nominal variables of the clinical examinations, including the existence of extension limitations in the elbow joint, pronation limitations in the elbow joint, supination limitation in the elbow joint, tenderness to touch on the radial head, tenderness to touch on ulnar head, tenderness to touch on the humerus epicondyle and hematoma development, with the prediction of radius head fracture that had been found appropriate in the radiographies. The logistic analyses of the data indicated that there is a significant relationship between the radius head fracture and the development of supination limitation in the elbow joint $(P=0.008)$, tenderness to palpation on radial head of the humerus $(P<0.001)$, tenderness to palpation on the ulnar head $(P=0.013)$ and tenderness to topical touch on epicondyle $(P=0.008)($ Table 1). 
Table 2: The relationship between the ulna head fracture and the clinical examination findings in patients with blunt elbow trauma

\begin{tabular}{clccc}
\hline Row & Clinical examination & OR & Cl (95\%) & P-value \\
\hline 1 & Extension limitation in elbow joint & 1.41 & $0.36-5.53$ & 0.610 \\
\hline 2 & Pronation limitation in elbow joint & 0.10 & $0.02-0.42$ & 0.002 \\
\hline 3 & Supination limitation in elbow joint & 13.31 & $1.78-99.62$ & 0.012 \\
\hline 4 & Tenderness to topical touch on radial head & 0.35 & $0.08-1.51$ & 0.160 \\
\hline 5 & Tenderness to topical touch on ulnar head & 0.05 & $0.01-0.2$ & $-?$ \\
\hline 6 & Tenderness to topical touch on epicondyle & - & 0.001 & 0.99 \\
\hline 7 & Development of hematoma in elbow joint & 0.35 & $0.09-1.29$ & 0.11 \\
\hline
\end{tabular}

Table 3: The relationship between the distal humerus fracture and the findings of clinical examinations of the patients with blunt elbow trauma

\begin{tabular}{clccc}
\hline Row & Clinical examination & OR & Cl (95\%) & P-value \\
\hline 1 & Extension limitation in elbow joint & 0.10 & $0.01-0.88$ & 0.03 \\
\hline 2 & Pronation limitation in elbow joint & 1.20 & $0.12-11.31$ & 0.87 \\
\hline 3 & Supination limitation in elbow joint & 0.30 & $0.03-2.58$ & 0.27 \\
\hline 4 & Tenderness to topical touch on radial head & 10.61 & $1.83-61.47$ & 0.008 \\
\hline 5 & Tenderness to topical touch on ulnar head & 2.06 & $0.18-22.74$ & 0.55 \\
\hline 6 & Tenderness to topical touch on epicondyle & 0.24 & $0.05-1.14$ & 0.07 \\
\hline 7 & Development of hematoma in elbow joint & 1.73 & $0.26-11.19$ & 0.56 \\
\hline
\end{tabular}

Table 4: The relationship between the proximal radius fracture and the findings of the clinical examinations in patients with blunt trauma fracture

\begin{tabular}{clccc}
\hline Row & Clinical examination & OR & Cl (95\%) & P-value \\
\hline 1 & Extension limitation in elbow joint & 0.13 & $0.01-1.13$ & 0.06 \\
\hline 2 & Pronation limitation in elbow joint & 1.19 & $0.10-13.51$ & 0.88 \\
\hline 3 & Supination limitation in elbow joint & 0.09 & $0.008-0.94$ & 0.04 \\
\hline 4 & Tenderness to topical touch on radial head & 0.68 & $0.18-2.51$ & 0.57 \\
\hline 5 & Tenderness to topical touch on ulnar head & 3.60 & $0.59-22.02$ & 0.16 \\
\hline 6 & Tenderness to topical touch on epicondyle & 0.50 & $0.12-2.01$ & 0.33 \\
\hline 7 & Development of hematoma in elbow joint & 0.44 & $0.10-1.87$ & 0.27 \\
\hline
\end{tabular}

Table 5: The relationship between the proximal ulna fracture and the findings of clinical examinations in patients with blunt elbow trauma

\begin{tabular}{clccc}
\hline Row & Clinical examination & OR & Cl (95\%) & P-value \\
\hline 1 & Extension limitation in elbow joint & 1.09 & $0.13-6.25$ & 0.92 \\
\hline 2 & Pronation limitation in elbow joint & 0.25 & $0.13-118.85$ & 0.42 \\
\hline 3 & Supination limitation in elbow joint & 6.83 & $0.005-3.99$ & 0.25 \\
\hline 4 & Tenderness to topical touch on radial head & 0.20 & $0.69-34.16$ & 0.11 \\
\hline 5 & Tenderness to topical touch on ulnar head & 0.30 & $0.26-42.15$ & 0.35 \\
\hline 6 & Tenderness to topical touch on epicondyle & 5.65 & $0.03-1.02$ & 0.05 \\
\hline 7 & Development of hematoma in elbow joint & 5.23 & $0.03-1.19$ & 0.07 \\
\hline
\end{tabular}

Next, the relationship between ulna head fracture with all these bimodal nominal variables was investigated. The logistic analysis of the date indicated that there is a significant relationship between ulna head fracture with such signs as pronation limitation in elbow joint $(P=0.002)$, supination limitation in the elbow joint $(P=0.012)$, tenderness to palpation on ulnar head $(P<0.001)$ (Table 2).

The logistic analysis of the data also showed that there is a significant relationship between the distal humerus fracture with extension limitation in elbow joint $(P=0.03)$ and tenderness to touch on radial head $(P=0.008)(P<0.05)($ Table 3$)$.

Furthermore, the logistic analysis of the data was indicative of a significant relationship between the proximal radius fracture with only supination limitation in elbow pit $(P=0.045)$ and it was not found having a significant relationship with the other studied clinical variables $(P>0.05)$ (Table 4).

Moreover, the logistic analysis of the data demonstrated that there is no significant relationship between the proximal ulna fracture with any of the clinical examination signs and, in the end, a significant relationship was found between the combined radioulnar fracture with such clinical examination sings as supination limitation in the elbow joint $(P=0.025)$ and hematoma development in the elbow joint $(P=0.009)(P<0.05)$ (Tables $5 \& 6$ ). 
Table 6: The relationship between combined humerus fractures with the findings of the clinical examinations in patients with blunt elbow trauma

\begin{tabular}{clccc}
\hline Row & Clinical examination & OR & Cl (95\%) & P-value \\
\hline 1 & Extension limitation in elbow joint & 1.43 & $0.05-8.66$ & 0.78 \\
\hline 2 & Pronation limitation in elbow joint & 0.35 & $0.07-112.35$ & 0.57 \\
\hline 3 & Supination limitation in elbow joint & 87.52 & $0.000-0.56$ & 0.02 \\
\hline 4 & Tenderness to topical touch on radial head & 0.86 & $0.12-10.35$ & 0.89 \\
\hline 5 & Tenderness to topical touch on ulnar head & 4.15 & $0.02-2.03$ & 0.19 \\
\hline 6 & Tenderness to topical touch on epicondyle & 1.49 & $0.08-5.32$ & 0.70 \\
\hline 7 & Development of hematoma in elbow joint & 14.32 & $0.01-0.51$ & 0.009 \\
\hline
\end{tabular}

\section{DISCUSSION}

Elbow injuries are the most common complaints for which the adults and children refer to the trauma emergency centers (12). There are standardized scales for determining the relationship between the clinical examinations regarding the injuries and fractures of knee and ankle for the time being and there have been performed researches from long ago concerning the applicability and importance of the clinical findings and the injuries expressions to consider the possibilities of fractures and dislocation and referral to radiographic tests for the precise diagnosis of the injuries incurred by knee and ankle. For instance, in a study conducted by Doctor Stiell in 1992, 32 standardized clinical variables like ecchymosis, limitation in range of motion, distension and local tenderness were evaluated for various sections of ankle such as interior and exterior malleolus, distal tibia and fibula as well as the other anatomic parts of the ankle in over 750 patients with blunt ankle trauma and injuries like falling down, sprain and in accident with vehicles by emergency medicine experts before referral to radiographic imaging and the information was inserted in special forms. Then, the patients were subjected to standard ankle and foot radiographies. Out of this number of the patients, 70 individuals had severe malleolar fracture and 32 individuals had severe mid-foot fractures. It was shown in data analysis that there is a significant difference between the existent factors assessable in clinical examinations like limitation in movement, loss of weight-bearing, local distension in various sides of the ankle such as interior and exterior malleolus and proximal fibula tenderness, exterior and interior malleolus in both of the patient groups with without severe malleolar fracture. This is while, no significant difference was found between the percentages of ecchymosis development, soft tissue tenderness and distention of anterior talofibular ligaments and anterior surface of the ankle in both of the groups with and without severe malleolar fracture (13). There are other similar studies performed in this regard and even there are cases found that the preliminary scales of the lower extremity injuries have been repeatedly evaluated (14-18).

But, a generally-accepted set of scales regarding the upper extremity fractures and injuries are yet to be produced according to the scientific researches we did in such information databases like Pubmed, Scopus and Google Scholar.

In 1991, Dr. Hawksworth published his findings regarding the elbow injuries. The study was the first of its type on the upper extremity injuries. It was conducted on 95 patients with elbow trauma and injuries. The entire patients were completely examined immediately after admission in triage then they were subjected to radiography. It was found in radiographs that 54 patients have serious injuries and fractures. Also, it had been pointed out in the post-admission examinations that out of the 95 referrals, 49 patients were incapable of performing elbow extensions. The analysis indicated that the sensitivity of extension inability has been $90.7 \%$ and only $9.3 \%$ had the chance of severe injuries and engagement from the individuals who could completely extend their elbow joints in the preliminary examinations (19).

In the study performed by Dr. Lennon et al, the results of which were published in 2007, 331 individuals, out of 407 patients referred for elbow injury and trauma, were subjected to radiographic evaluations. Four scales, i.e. complete extension and flexion of elbow joint, the ability to perform pronation/supination and normal range of motion, were evaluated by the physician. The data results indicated that these four clinical scales, complete extension and flexion of elbow joint, the ability to perform pronation/supination and normal range of motion, are $91 \%, 77 \%, 79 \%$ and $97 \%$, respectively, for all of the studied adults and children and this is reflective of these four scales' high specificity and also suggestive of the idea that one of the abovementioned scales of the patients would be definitely abnormal if irregularities were documented in the radiographs. But, the sensitivities of these clinical scales were found lower in this study and values equal to $47 \%, 56 \%, 50 \%$ and $21 \%$ were calculated for sensitivity rates of complete extension and flexion of elbow joint, the ability to perform pronation/supination and normal range of motion, respectively. Such a low sensitivity means that the patients whose results were found normal in radiographic imaging could have also demonstrated unusual signs in the examinations (20). 
Then, in 2008, the study results by Dr. Appelboam were published. The study evaluated the complete extension rates of the elbow joints in 2127 adults and children who had referred with elbow joint trauma and injuries during a two-year period. The study aimed at investigating and assessing extension rate as an effective clinical scale for rejecting the joint fracture likelihood. The elbow extension rates of the entire individuals who had referred to the treatment center were measured in the above study and then the entire patients underwent diagnostic radiography. The information obtained for 1740 individuals qualified to enter the study was evaluated and all of them were referred to radiography department. Out of the foresaid number, 602 individuals could exhibit complete elbow extension in the clinical tests and 1138 individuals could not perform it completely. Out of these latter 1138 individuals, 521 individuals were diagnosed with joint fractures. Also, the radiographic investigations showed that 17 out of the 602 individuals who had been able to perform complete elbow extensions have fractures. Next, sensitivity and specificity and the positive and negative predictive values of the clinical examinations for both of the adult and child groups as well as in total were measured. Based on the study, the sensitivity and specificity rates of complete elbow extension tests in only the adult group for the prognostication of the fracture were $98.4 \%$ and $47.7 \%$, respectively; also, it was found out that the sensitivity and specificity rates of the fractures along with joint effusions are $97.3 \%$ and $54.3 \%$, respectively (21). In fact, the results obtained in this study are a lot different from the results put forth by Dr. Lennon because only the specificity of extension rates was only higher in the latter study and the sensitivity value was lower. One possible reason giving rise to such a large difference might be the study sample volume and some of the other study entrance scales. The individuals who were not deemed in need of radiography were put aside from data analysis in the study performed by Lennon and the results have only been calculated for those for whom radiographs had been requested (20). However, it seems that the foresaid differences could not be as accountable.

In the present study, as well, the results obtained for the children were also investigated. The results indicated that the sensitivity rates of the test in prognosticating only fracture as well as fracture and effusion were $94.6 \%$ and $93.7 \%$, respectively. The specificity of the test for the prognostication of only fracture was $49.5 \%$ which was found increased to $54.8 \%$ in prognosticating fracture or effusion. In an investigation of the totals and disregarding the age, the data analysis also indicated that the extension test has a sensitivity rate and a specificity rate of $96.8 \%$ and $48.5 \%$, respectively, for the prognostication of only fracture. If the diagnosis objective is turned to effusion or fracture in the joint, the extension test's sensitivity and specificity will become $95.8 \%$ and $54.6 \%$, respectively (21). The values obtained herein for specificity and sensitivity of the extension test are still indicative of a large difference with the results obtained in the study by Lennon (4). It seems that the ability or inability to perform extension is enumerated as an important evaluation in the clinical examinations. Due to the same reason, extension is a valuable factor in a great many of the studies and it is usually reported. But, the entire joint examinations, i.e. complete extension and flexion of elbow joint, the ability to perform pronation/supination and normal range of motion, have been evaluated in the study by Dr. Lennon which makes it more similar to our study in this regard. We, as well, besides the extension, considered other scales of joint movements like pronation and supination in the evaluations.

In continuation to the studies performed in this area, another study showed up. It was the research by Dr. Baker et al which was conducted in 2010 specifically aiming at evaluation of elbow injuries in 177 children ranging in age from 3 to 6 years of age. The elbow joint's range of motion, including extension, flexion, pronation and supination, was investigated and examined in the individuals who had referred for elbow injuries. Then, the children were subjected to radiographies. In the radiographic results of the children, 175 irregularities were totally diagnosed, including 75 fractures and 2 joint dislocations. Effusion was the only injury evidenced in 30 of these children. It had been found in the preliminary examinations that 106 children generally had unusual and disrupted range of motion and the test's sensitivity and specificity were found $93.4 \%$ and $33.8 \%$, respectively (22).

The disorders in extension and flexion were evaluated in a more specialized analysis and also the sensitivity and specificity values of the test were calculated. The results indicated that the sensitivity and specificity of the flexion disorder, as a prognosticative clinical factor, are equal to $88.7 \%$ and $45.1 \%$, respectively. Moreover, the sensitivity and specificity of the extension disorder test were found equal to $80.2 \%$ and $64.8 \%$. Also, the physicians, in this study, were asked during the preliminary examinations of the patients to assert their clinical judgment of the existence or absence of serious elbow injury to see if it can be used as a prognostication scale. The results indicated that the physicians' clinical judgment regarding serious elbow joint injury enjoy sensitivity and specificity rates equal to $90.6 \%$ and $54.3 \%$, respectively (22).

It can be stated in a comparison of these researches with the study we carried out that our study not only dealt with the investigation of joint movement disorders in the elbow joint injuries but also the other examinations as well as the 
soft tissue injury likelihoods were also evaluated because we believe that although extension test can be employed to come to a clinical conclusion in certain emergency situations and due to its acceptable sensitivity, the other important and significant points can also contribute to the reduction of the number of false diagnoses during the preliminary examinations. On the other hand, although the scale reported in the aforementioned studies was mostly based on specificity and sensitivity and, of course, it is of a great value in selection of test type, we believe that the use of logistic regression test is a more precise statistical method for performing investigations regarding various types of fractures and their relationships with the developed sings as a result of which more precious information can be acquired.

\section{CONCLUSION}

Generally, although the number of sample volumes used by various studies have been different and also with the existence of different clinical scales in investigating the extent and type of the possible injuries in blunt (non-penetrating) elbow trauma, it seems that the joint movement evaluations, especially joint extension, enjoys a high sensitivity, to wit the high sensitivity of any disorders in extension, flexion, pronation and supination in elbow joint, whether completely or partly, can be indicative of the test power in injury prognostication. But, unfortunately, the majority of these tests lack a high sensitivity as a result of which the number of false diagnosis will increase. But, in the study we performed herein based on a more different method, the fracture type was investigated and analyzed in separate according to the type of the clinical findings during the preliminary examinations and this indicated that there is a significant relationship between the radius head fracture with limitation in performing such movements as supination, tenderness to topical touch on radial head, tenderness to topical touch on ulnar head and tenderness to touch on humerus epicondyle; in addition, the data analyses also indicated that there is a significant relationship between the ulna head fracture with limitations in such motions as pronation, supination and tenderness to touch on ulna head fracture.

Also, a significant relationship was documented between the distal humerus fracture with such clinical signs as limitation in extension and tenderness to topical touch on radial head.

In the end, the logistic data analysis indicated that there is a significant relationship between proximal radius head fracture only with such a clinical symptom as limitation in supination in elbow joint and that there is also a significant relationship between combined radioulnar fracture in the elbow joint with supination limitation and hematoma development in elbow joint, but no significant relationship was found between the proximal ulna fracture with any of the studied clinical signs based on the data analysis.

\section{REFERENCES}

1. Chen NC. Elbow Fractures with Instability: Evaluation and Treatment Strategies. Hand Clin. 2018;34(1):75-83. https://doi.org/10.1016/j.hcl.2017.09.008 PMid:29169599

2. Nanno M, Sawaizumi T, Ito H. Transverse divergent dislocation of the elbow with ipsilateral distal radius fracture in a child. J Orthop Trauma. 2007;21(2):145-9. https://doi.org/10.1097/BOT.0b013e318032c4be PMid:17304072

3. Siebenlist S, Braun KF. Elbow dislocation fractures. Unfallchirurg. 2017;120(7):595-610. https://doi.org/10.1007/s00113-017-0373-7 PMid:28664232

4. Ahmad R, Ahmed SM, Annamalai S, Case R. Open dislocation of the elbow with ipsilateral fracture of the radial head and distal radius: a rare combination without vascular injury. BMJ Case Rep. 2009;2009:bcr2006044016.

5. Sakata J, Nakamura E, Suzuki T, Suzukawa M, Akaike A, Shimizu K, Hirose N. Efficacy of a Prevention Program for Medial Elbow Injuries in Youth Baseball Players. Am J Sports Med. 2017:363546517738003.

6. Sato R, Hamada Y, Hibino N, Nishisho T, Tonogai I, Endo K, et al. Restoration of the Active Forearm Rotation Using Vascularized Free Fibular Graft and Radial Head Arthroplasty for a Wide Defect of the Proximal Radius. J Hand Surg Asian Pac Vol. 2017;22(4):531-534. https://doi.org/10.1142/S0218810417720406 PMid:29117845

7. Yoshida M, Goto H, Takenaga T, Tsuchiya A, Sugimoto K, Musahl V, et al. Anterior and posterior bands of the anterior bundle in the elbow ulnar collateral ligament: ultrasound anatomy. J Shoulder Elbow Surg. 2017;26(10):1803-1809. https://doi.org/10.1016/j.jse.2017.05.025 PMid:28735845

8. Bazzocchi A, Aparisi Gómez MP, Bartoloni A, Guglielmi G. Erratum: Emergency and Trauma of the Elbow. Semin Musculoskelet Radiol. 2017;21(5):649-650. https://doi.org/10.1055/s-0037-1606338 PMid:29025193

9. Dwek JR. A Segmental Approach to Imaging of Sports-Related Injuries of the Pediatric Elbow. Sports Health. 2012;4(5): 442-452. https://doi.org/10.1177/1941738112448733 PMid:23016119 PMCid:PMC3435948 
10. Capo JT, Shamian B, Francisco R, Tan V, Preston JS, Uko L, et al. Fracture pattern characteristics and associated injuries of high-energy, large fragment, partial articular radial head fractures: a preliminary imaging analysis. J Orthop Traumatol. 2015;16(2):125-31. https://doi.org/10.1007/s10195-014-0331-x PMid:25542062 PMCid:PMC4441642

11. Russell DW, Janz DR, Emerson WL, May AK, Bernard GR, Zhao Z, et al. Early exposure to hyperoxia and mortality in critically ill patients with severe traumatic injuries. BMC Pulm Med. 2017;17(1):29. https://doi.org/10.1186/s12890-017-0370-1 PMid:28158980 PMCid:PMC5291954

12. Freed HA, Shields NN. Most frequently overlooked radiographically apparent fractures in a teaching hospital emergency department. Annals of emergency medicine. 1984;13(10):900-4. https://doi.org/10.1016/S01960644(84)80666-6

13. Stiell IG, Greenberg GH, McKnight RD, Nair RC, McDowell I, Worthington JR. A study to develop clinical decision rules for the use of radiography in acute ankle injuries. Annals of emergency medicine. 1992;21(4):384-90. https://doi.org/10.1016/S0196-0644(05)82656-3

14. Shell IG, Greenberg GH, McKnight RD, Nair RC, McDowell I, Reardon M, et al. Decision rules for the use of radiography in acute ankle injuries: refinement and prospective validation. JAMA. 1993;269(9):1127-32. https://doi.org/10.1001/jama.1993.03500090063034

15. Stiell IG, Greenberg GH, Wells GA, McDowell I, Cwinn AA, Smith NA, et al. Prospective validation of a decision rule for the use of radiography in acute knee injuries. Jama. 1996;275(8):611-5. https://doi.org/10.1001/jama.1996.03530320035031 PMid:8594242

16. Emparanza JI, Aginaga JR. Validation of the Ottawa knee rules. Annals of emergency medicine. 2001;38(4):3648. https://doi.org/10.1067/mem.2001.118011 PMid:11574791

17. Stiell IG, Greenberg GH, McKnlght RD, Nalr RC, McDowell I, Reardon M, et al. Decision rules for the use of radiography in acute ankle injuries. health care. 1993;13:15.

18. Bulloch B, Neto G, Plint A, Lim R, Lidman P, Reed M, et al. Validation of the Ottawa Knee Rule in children: a multicenter study. Annals of emergency medicine. 2003;42(1):48-55. https://doi.org/10.1067/mem.2003.196 PMid:12827123

19. Hawksworth $C$, Freeland $P$. Inability to fully extend the injured elbow: an indicator of significant injury. Archives of emergency medicine. 1991;8(4):253-6. https://doi.org/10.1136/emj.8.4.253 PMid:1772537 PMCid:PMC1285793

20. Lennon RI, Riyat MS, Hilliam R, Anathkrishnan G, Alderson G. Can a normal range of elbow movement predict a normal elbow x ray? Emergency medicine journal. 2007;24(2):86-8. https://doi.org/10.1136/emj.2006.039792 PMid:17251609 PMCid:PMC2658213

21. Appelboam A, Reuben A, Benger J, Beech F, Dutson J, Haig S, et al. Elbow extension test to rule out elbow fracture: multicentre, prospective validation and observational study of diagnostic accuracy in adults and children. BMJ. 2008;337:a2428. https://doi.org/10.1136/bmj.a2428 PMid:19066257 PMCid:PMC2600962

22. Baker M, Borland M. Range of elbow movement as a predictor of bony injury in children. Emerg Med J. 2011;28(8):666-9. https://doi.org/10.1136/emj.2010.091124 PMid:20713361

$\diamond \diamond \diamond \diamond \diamond \diamond \diamond$

http://www.ejgm.co.uk 